\title{
Collaborative Filtering Using Restoration Operators
}

\author{
Atsuyoshi Nakamura, Mineichi Kudo, and Akira Tanaka \\ Division of Systems and Information Engineering \\ Graduate School of Engineering \\ Hokkaido University, Sapporo 060-8628 Japan \\ \{atsu,mine, takira\}@main.eng.hokudai.ac.jp
}

\begin{abstract}
We propose a new collaborative filtering method that uses restoration operators. The problem of restoration by operators was originally studied in the field of digital image restoration [9]. We also consider the problem of selecting items that users should be asked to rate in order to achieve a small expected squared error, and we propose a greedy method as a solution of this problem. According to our experimental results, prediction performance of restoration operators is good when the number of observed ratings is small, and our greedy method outperforms random query item selection.
\end{abstract}

\section{Introduction}

Information filtering has become an important technology in recent years due to wide spread of Internet. This technology enables systems to learn a user's personal preference, and recommend items (such as news articles, music and books) that are preferred by the user. Collaborative filtering, an information filtering technique, has been studied extensively in recent years $[10,11,2,8]$. This filtering technique enables a system to recommend items to a user that are preferred by similar users. Generally, a user's preference for an item is represented by a rating, and calculation of similarities between users and judgement on whether similar users prefer or not are based on the ratings. Collaborative filtering problem can be also considered as the problem of predicting unknown entry values from known entry values of a partially known user-item rating matrix.

The performance of a system in giving initial recommendations for a new user is important because a user will not continue to use the system if it takes long time for the system to learn the user's preference. In this paper, we propose a collaborative filtering method using restoration operators that can make good predictions based on a small number of ratings. Furthermore, we consider the problem of selecting items that a new user is asked to rate in order to achieve optimal prediction performance. This kind of learning, in which a learner obtains information needed to learn actively, is called active learning [4] and it is one of the current hot research topics.

There have been a few studies on collaborative filtering using active learning. Goldberg et al. [7] developed a system that asks new users to rate the same set of 
items called the gauge set. The system uses the ratings for the items in the gauge set to classify the user into one of the clusters and then recommends the items that are popular among the users belonging to that cluster. However, Goldberg et al. did not describe how the gauge set is selected. Boutiler and Zemel [3] proposed a system that asks new users to rate an item with the maximum expected value of information with respect to the current probabilistic model. Dasgupta et al. [5] considered that there are a number of typical users and that each user's ratings are close to those of one typical user. They proposed a query selection algorithm and analyzed the number of queries to an arbitrary user needed for finding a typical user with similar ratings. Here, a query means asking a user to rate a given item. Query selection, therefore, means determination of which item is given.

Restoration operators have been extensively studied in the field of digital image restoration $[1,9]$. For a known degration operator $P$, they have studied the problem of how to restore a digital image $\boldsymbol{x} \in \Re^{m}$ from a given degraded image $P \boldsymbol{x} \in \Re^{k}(k<m)$. Collaborative filtering problem can be also seen as a restoration problem in which a vector $\boldsymbol{x}$ of user's preference for all items is restored from a partial vector $P \boldsymbol{x}$ of $\boldsymbol{x}$, where $P$ is an operator that restricts the components to those having observed values. Considering characteristics of collaborative filtering problem, we propose a collaborative filtering method using an unbiased Wiener filter without additive noise. Our experimental results showed that our method outperforms the correlation-based method with the best performance when the number of observed ratings is small.

In our problem setting, the active learning problem, the problem of selecting the best items that a new user is asked to rate, is that of finding a restriction operator $P$ that minimizes the expected squared error. However, to the best of our knowledge, there are no efficient methods to find the best operator $P$ among $O\left(m^{k}\right)$ operators, where $m$ is the number of items and $k$ is the number of queries. Instead of finding the best operator, we propose a greedy method that finds an operator with small expected error by greedy search which involves comparison of $O(\mathrm{~km})$ operators. Our experimental results showed that our greedy method is better than random selection in terms of prediction performance.

This paper is organized as follows. In Section 2, we formalize a collaborative filtering problem as a restoration problem and show its solution. In Section 3, we describe an optimal query item selection problem and our greedy selection method. Results of experiments using the EachMovie data set are shown in Section 4, and future work is discussed in Section 5.

\section{Collaborative Filtering Problem as a Restoration Problem}

Let $m$ denote the number of items and $\boldsymbol{x} \in \Re^{m}$ denote a vector of the user's preference for all items. Assume that $\boldsymbol{x}$ is generated according to an arbitrary distribution over $\Re^{m}$. The collaborative filtering task can be regarded as a restoration of vector $\boldsymbol{x}$ from a given partial vector of $\boldsymbol{x}$. Such a restoration problem has 
been studied in the area of digital image restoration. In this section, we formalize a collaborative filtering problem like the formalization of a digital image restoration problem presented in [9].

For $J=\left\{i_{1}, i_{2}, \ldots, i_{k}\right\} \subseteq\{1,2, \ldots, m\}$, let $P_{J}$ denote a restriction transformation (matrix) that restricts components of a vector to those in $J$, namely, $P_{J}$ is a transformation such that $P_{J} \boldsymbol{x}=\left(x_{i_{1}}, x_{i_{2}}, \ldots, x_{i_{k}}\right)^{T}$ for $\boldsymbol{x}=\left(x_{1}, x_{2}, \ldots, x_{m}\right)^{T}$. Observing $P_{J} \boldsymbol{x}$ for some $J$, we want to find an estimation $\hat{\boldsymbol{x}}$ of $\boldsymbol{x}$ which is close to $\boldsymbol{x}$, that is, the mean squared error (MSE) $\|\boldsymbol{x}-\hat{\boldsymbol{x}}\|^{2}$ is as small as possible, where $\|\cdot\|$ denotes Euclidean norm. Since $\hat{\boldsymbol{x}}$ is calculated by applying a restoration transformation to $P_{J} \boldsymbol{x}$, this problem is reduced to that of finding an optimal transformation. For fixed $J$, this problem can be formalized as follows if restoration transformations are restricted to affine transformations.

Problem 1. For given $J$ with $^{1}|J|=k$, find $\left(B_{\text {opt }}, \boldsymbol{w}_{\text {opt }}\right)$ satisfying

$$
\left(B_{\mathrm{opt}}, \boldsymbol{w}_{\mathrm{opt}}\right)=\arg \min _{(B, \boldsymbol{w})} E_{\boldsymbol{x}}\left\|\boldsymbol{x}-B P_{J} \boldsymbol{x}-\boldsymbol{w}\right\|^{2},
$$

where the minimization is with respect to all possible linear transformations $B: \Re^{k} \rightarrow \Re^{m}$ and all possible vectors $\boldsymbol{w} \in \Re^{m}$.

This problem setting can be regarded as that of an unbiased Wiener filter without additive noise. (See [9].) The solution for this problem is as follows. Note that $\cdot^{T}$ and $\operatorname{tr}(\cdot)$ denote the transpose and trace of a matrix, respectively.

Solution 1.

$$
\begin{aligned}
& B_{\mathrm{opt}}=R P_{J}^{T}\left(P_{J} R P_{J}^{T}\right)^{+}, \\
& \boldsymbol{w}_{\mathrm{opt}}=\left(I-B_{\mathrm{opt}} P_{J}\right) E_{\boldsymbol{x}} \boldsymbol{x} \text { and } \\
& \min _{(B, \boldsymbol{w})} E_{\boldsymbol{x}}\left\|\boldsymbol{x}-B P_{J} \boldsymbol{x}-\boldsymbol{w}\right\|^{2}=\operatorname{tr}\left(R-B_{\mathrm{opt}} P_{J} R\right),
\end{aligned}
$$

where $R=E_{\boldsymbol{x}}\left(\boldsymbol{x}-E_{\boldsymbol{x}} \boldsymbol{x}\right)\left(\boldsymbol{x}-E_{\boldsymbol{x}} \boldsymbol{x}\right)^{T}$ and $\left(P_{J} R P_{J}^{T}\right)^{+}$is the Moore-Penrose inverse of $P_{J} R P_{J}^{T}$.

Why do not we take noise into account? In digital image restoration, we assume that an original image $\boldsymbol{x}$ contains no noise and that as training data we observe $P \boldsymbol{x}+\boldsymbol{n}$, an image which is degraded by a degradation operator $P$ and to which noise $\boldsymbol{n}$ is added, as well as $\boldsymbol{x}$. If we take noise into account in our collaborative filtering problem, it should be assumed that we observe a user's preference $\boldsymbol{x}$ that contains noise and a partial vector $P_{J} \boldsymbol{x}$ that does not contain additional noise. Assume that the observed vector $\boldsymbol{x}$ contains noise $\boldsymbol{n}$. Then, what we want to estimate is $\hat{\boldsymbol{x}}$ that minimizes $\|\boldsymbol{x}-\boldsymbol{n}-\hat{\boldsymbol{x}}\|^{2}$. Thus, instead of Equation (1), we should use the equation

$$
\left(B_{\mathrm{opt}}, \boldsymbol{w}_{\mathrm{opt}}\right)=\arg \min _{(B, \boldsymbol{w})} E_{\boldsymbol{x}} E_{\boldsymbol{n}}\left\|\boldsymbol{x}-\boldsymbol{n}-B P_{J} \boldsymbol{x}-\boldsymbol{w}\right\|^{2} .
$$

If we assume that $\boldsymbol{x}$ and $\boldsymbol{n}$ are mutually independent and that $E(\boldsymbol{n})=\mathbf{0}$,

$$
E_{\boldsymbol{x}} E_{\boldsymbol{n}}\left\|\boldsymbol{x}-\boldsymbol{n}-B P_{J} \boldsymbol{x}-\boldsymbol{w}\right\|^{2}=E_{\boldsymbol{x}}\left\|\boldsymbol{x}-B P_{J} \boldsymbol{x}-\boldsymbol{w}\right\|^{2}+E_{\boldsymbol{n}}\|\boldsymbol{n}\|^{2} .
$$

\footnotetext{
${ }^{1}$ Here, $|J|$ denotes the number of elements in $J$.
} 
Since the term $E_{\boldsymbol{n}}\|\boldsymbol{n}\|^{2}$ does not affect the minimization, Equation (2) coincides with Equation (1). Therefore, our problem setting deals with the case in which noise is contained in a user's preference, which can be inevitable because user's evaluation values are quantized.

When Solution 1 is used, consideration must be given to the method used for estimating the covariance matrix $R=E_{\boldsymbol{x}}\left(\boldsymbol{x}-E_{\boldsymbol{x}} \boldsymbol{x}\right)\left(\boldsymbol{x}-E_{\boldsymbol{x}} \boldsymbol{x}\right)^{T}$. What makes this difficult is that this estimation must be done from the user's preference dataset $X$, the elements of which have many missing values. Here, we estimate $R$ as follows. Let $n_{i}$ denote the number of elements in $X$ whose $i$ th component is not missing. Let $\hat{\bar{x}}=\left\{\hat{\bar{x}}_{1}, \hat{\bar{x}}_{2}, \ldots, \hat{\bar{x}}_{m}\right\}$ denote an estimation of $E_{\boldsymbol{x}} \boldsymbol{x}$. Then, in our estimation,

$$
\hat{\bar{x}}_{i}=\sum_{x \in X, x_{i} \neq *} x_{i} / n_{i},
$$

where $*$ denotes the missing value. For $\boldsymbol{x} \in X$, let $\boldsymbol{x}^{\prime}$ denote the vector that is made from $\boldsymbol{x}$ by replacing all missing values $x_{i}$ with $\hat{\bar{x}}_{i}$. Our estimation $\hat{r}_{i, j}$ of the $(i, j)$ entry value of $R$ is calculated as

$$
\hat{r}_{i, j}=\frac{1}{n} \sum_{\boldsymbol{x} \in X}\left(x_{i}^{\prime}-\hat{\bar{x}}_{i}\right)\left(x_{j}^{\prime}-\hat{\bar{x}}_{j}\right),
$$

where $n$ is the number of elements in $X$.

\section{Optimal Query Item Selection}

Another concern is for which items we should know the user's preference in order to estimate the whole vector of the user's preference as precisely as possible. The problem of optimal query item selection is that of finding the best $J$, which is fixed in Problem 1.

Problem 2. For given $1 \leq k \leq n$, find $J_{\text {opt }} \subseteq\{1,2, \ldots, m\}$ satisfying

$$
J_{\mathrm{opt}}=\arg \min _{J:|J|=k} \min _{(B, \boldsymbol{w})} E_{\boldsymbol{x}}\left\|\boldsymbol{x}-B P_{J} \boldsymbol{x}-\boldsymbol{w}\right\|^{2},
$$

where the second minimization is with respect to all possible linear transformations $B: \Re^{k} \rightarrow \Re^{m}$ and all possible vectors $\boldsymbol{w} \in \Re^{m}$.

By plugging Solution 1 into Equation (4), we obtain the following equation:

$$
J_{\text {opt }}=\arg \max _{J:|J|=k} \operatorname{tr}\left(R P_{J}^{T}\left(P_{J} R P_{J}^{T}\right)^{+} P_{J} R\right) .
$$

Finding $J_{\text {opt }}$ requires calculation of $\operatorname{tr}\left(R P_{J}^{T}\left(P_{J} R P_{J}^{T}\right)^{+} P_{J} R\right)$ for $m(m-$ $1) \cdots(m-k+1) / k$ ! combinations $J$ of items, so it is not practical for a large value of $m$. In that case, we use a greedy method by which optimal items are added to $J$ one by one until the number of items in $J$ becomes $k$. Our greedy method uses $J_{k}$ instead of $J_{\text {opt }}$. $J_{k}$ is defined as follows:

$$
J_{k}=\arg \max \left\{\operatorname{tr}\left(R P_{J}^{T}\left(P_{J} R P_{J}^{T}\right)^{+} P_{J} R\right): J \subset J_{k-1},|J|=k\right\}, J_{0}=\emptyset .
$$

Note that the number of combinations $J$ in calculation of $J_{k}$ is $O(k m)$, while that in calculation of $J_{\mathrm{opt}}$ is $O\left(\mathrm{~m}^{k}\right)$. 


\section{Experiments}

We conducted two experiments: one for evaluation of prediction performance based on a small number of rated items, and the other for evaluation of query item selection by our greedy method.

\subsection{Correlation-Based Methods}

We compared the performance of our method to the performances of correlationbased methods $[10,11]$. By correlation-based methods, predictions are made using correlation coefficients between each pair of users that are calculated from ratings for the commonly rated items. The correlation coefficient between users $\boldsymbol{x}$ and $\boldsymbol{y}$ is calculated as follows:

$$
w_{\boldsymbol{x}, \boldsymbol{y}}=\frac{\sum_{x_{j} \neq *, y_{j} \neq *}\left(x_{j}-c_{\boldsymbol{x}, j}\right)\left(y_{j}-c_{\boldsymbol{y}, j}\right)}{\sqrt{\sum_{x_{j} \neq *, y_{j} \neq *}\left(x_{j}-c_{\boldsymbol{x}, j}\right)^{2} \sum_{x_{j} \neq *, y_{j} \neq *}\left(y_{j}-c_{\boldsymbol{y}, j}\right)^{2}}},
$$

where $c_{\boldsymbol{x}, j}$ is a center value for $\operatorname{user}^{2} \boldsymbol{x}$ and item $j$. Prediction of $x_{i}$ for user $\boldsymbol{x}$ with $x_{i}=*$ are made using correlation coefficients $w_{\boldsymbol{x}, \boldsymbol{y}}$ between user $\boldsymbol{x}$ and other users $\boldsymbol{y}$ with $y_{i} \neq *$ as follows:

$$
x_{i}=c_{\boldsymbol{x}, i}+\frac{\sum_{y_{i} \neq *} w_{\boldsymbol{x}, \boldsymbol{y}}\left(y_{i}-c_{\boldsymbol{y}, i}\right)}{\sum_{y_{i} \neq *}\left|w_{\boldsymbol{x}, \boldsymbol{y}}\right|} .
$$

With respect to $c_{\boldsymbol{x}, i}$, we consider the following three variations.

1. Fixed center

In this variation, $c_{\boldsymbol{x}, i}$ is the same constant for all $\boldsymbol{x}$. The method using this correlation coefficient is called constrained Pearson $r$ algorithm in [11].

2. User mean

In this variation, $c_{\boldsymbol{x}, i}$ is the average of $x_{j}$ for all item $j$ with $x_{j} \neq *$. The method using this correlation coefficient is called Pearson $r$ algorithm in [11]. In the case of new users having no ratings, we use the average of all scores rated by all users.

3. Item mean

In this variation, $c_{\boldsymbol{x}, i}$ is the average of $x_{i}$ for all users $\boldsymbol{x}$ with $x_{i} \neq *$, namely, $c_{\boldsymbol{x}, i}=\hat{\overline{\boldsymbol{x}}}$, where $\hat{\overline{\boldsymbol{x}}}$ is defined in Equation (3).

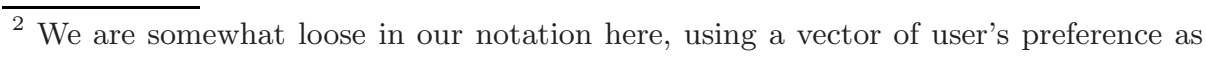
if it were the user itself. 


\subsection{Data}

In our experiment, we used the EachMovie collaborative filtering data set [6]. The data set consists of 2,811,983 numeric ratings for 1,628 movies evaluated by 72,916 users. The numeric rating for a pair of user and movie represents how much the user likes the movie on a six-point scale $(0.0,0.2,0.4,0.6,0.8,1.0)$. Note that only $2.37 \%$ of the user-movie matrix is filled.

Table 1. Statistical information on the data used in our experiments

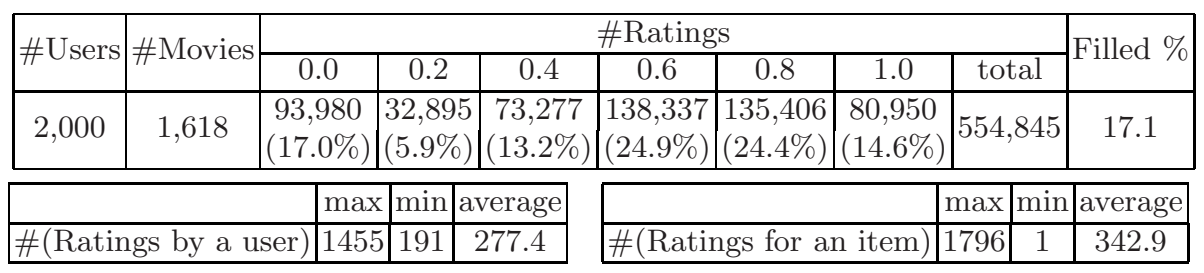

In our experiments, We used the ratings of 2,000 users with the largest number of ratings. Statistical information on our data is shown in Table 1. Note that $17.1 \%$ of the user-movie matrix of the data is filled.

\subsection{Performance Measures}

We evaluate the performance by two measures: mean squared error and recallprecision curve. The former is suitable to our problem setting, but the latter is more appropriate from a practical point of view. The following is an explanation of the method used for drawing recall-precision curves.

Rating values are divided into two classes, hot $(0.8$ or 1.0$)$ and cold $(0.0,0.2$, 0.4 and 0.6$)$, as in [2]. Precision and recall are used to evaluate what degree a method correctly predicts a set of hot movies for each user. Let $T$ denote the set of hot movies for an arbitrary user and $S$ denote the set of movies predicted to be hot for that user. Then, precision and recall for this prediction is defined as follows:

$$
\text { precision }=\frac{|T \cap S|}{|S|} \quad \text { recall }=\frac{|T \cap S|}{|T|} .
$$

For methods in which prediction values are given by real numbers, the set of movies predicted to be hot can be obtained by determining a threshold and selecting movies whose predicted values are greater than the threshold. In such cases, a recall-precision curve can be drawn by moving the threshold. We want to know the performance averaged over all users, but the problem is the difference in user's thresholds for the same recall. Thus, for recall $r$ and user $u$, we find the set $S_{r, u}$ of movies predicted to be hot by lowering the threshold until recall $r$ is achieved, and we calculate averaged precision for recall $r$ as follows:

$$
\text { averaged precision for recall } r=\frac{\sum_{u \in U}\left|T_{u} \cap S_{r, u}\right|}{\sum_{u \in U}\left|S_{r, u}\right|},
$$


where $T_{u}$ is the set of hot movies for user $u$ and $U$ is the set of users. We draw the recall-precision curve by moving recall $r$.

\subsection{Experimental Methodology}

We randomly divided the 2,000 users into 10 groups and carried out a 10-fold cross validation. We first estimated the covariance matrix $R$ using only training data. Then, for each user in a test data, we selected a set of movies for queries from the movies whose scores are known. Based on the ratings for the selected movies, predictions for the other movies whose scores are known were made. We evaluated methods by prediction performance averaged over all users. In the case of random query item selection, prediction performance was also averaged over five runs.

\subsection{Results}

Prediction Performance Based on a Small Number of Rated Items. We compared prediction performance of restoration operators with prediction performances of correlation-based methods when a small number of items are selected randomly as a set of items that are assumed to be rated.

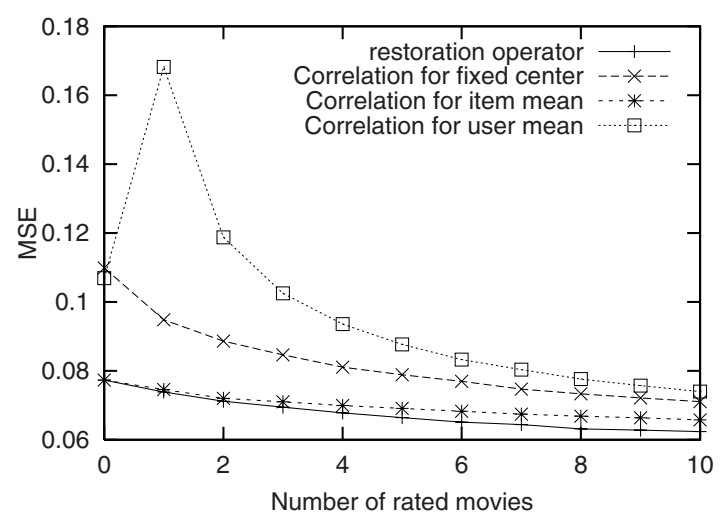

Fig. 1. Learning curves

Fig. 1 shows the relation between the number of rated movies and MSE. Here, predictions by restoration operators using no rating are those by the estimated mean vector $\hat{\overline{\boldsymbol{x}}}$ defined by Equation (3). Among the correlation-based methods, the method using the item mean performed best. As can be seen in Fig. 1, prediction performance of restoration operators was better than prediction performances of correlation-based methods.

Fig. 2 shows recall-precision curves when the number of rated movies are five and ten. The results are consistent with the results in terms of MSE. Prediction performances of two correlation-based methods using the fixed center 

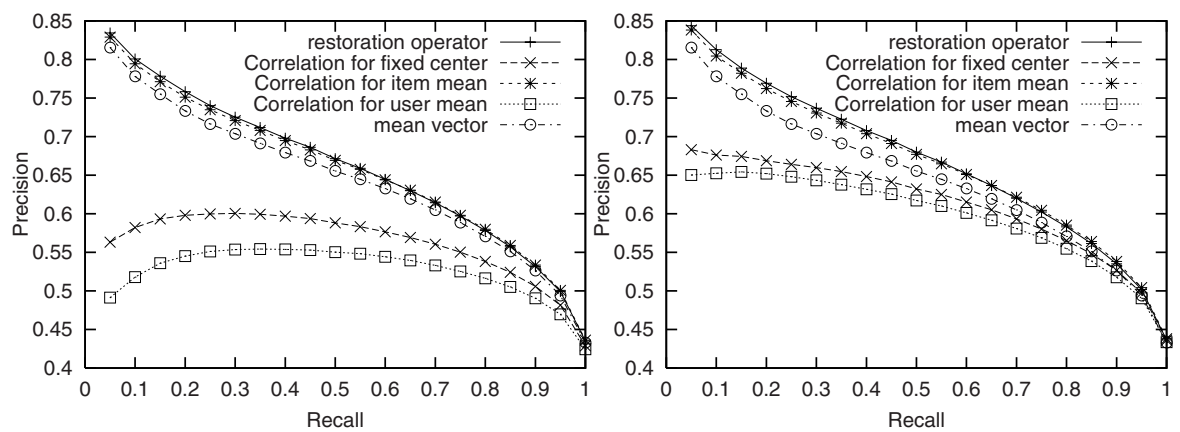

Fig. 2. Recall-precision curves (Left: prediction using 5 rated movies, Right: prediction using 10 rated movies)

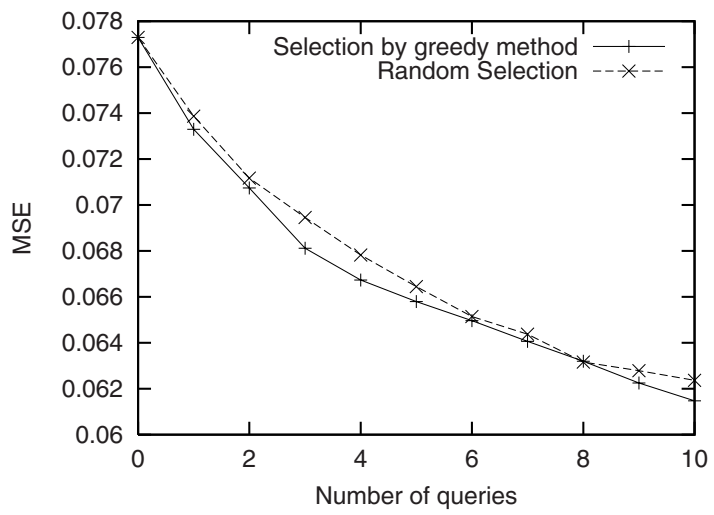

Fig. 3. Learning curves

and the user mean could not even exceed prediction performance of the mean verctor. The method using restoration operators slightly outperformed the best correlation-based method using the item mean at low recalls, which are more important parts because recommender systems only present high-ranking items.

Effectiveness of Query Item Selection. We investigated the effectiveness of our greedy query item selection.

Fig. 3 shows the relation between the number of queries and MSE. AS can be seen in the figure, MSE decreases as the number of queries increases. We compared prediction performance of our greedy methhod with that of random selection. The graph shows the greedy method outperformed random selection.

The left panel of Fig. 4 shows recall-precision curves for predictions made by the greedy method and the mean vector. It can be seen that the perfomance improves as the number of queries increases, particularly at low recalls. Improvement in performance at low recalls with respect to number of queries is shown in 

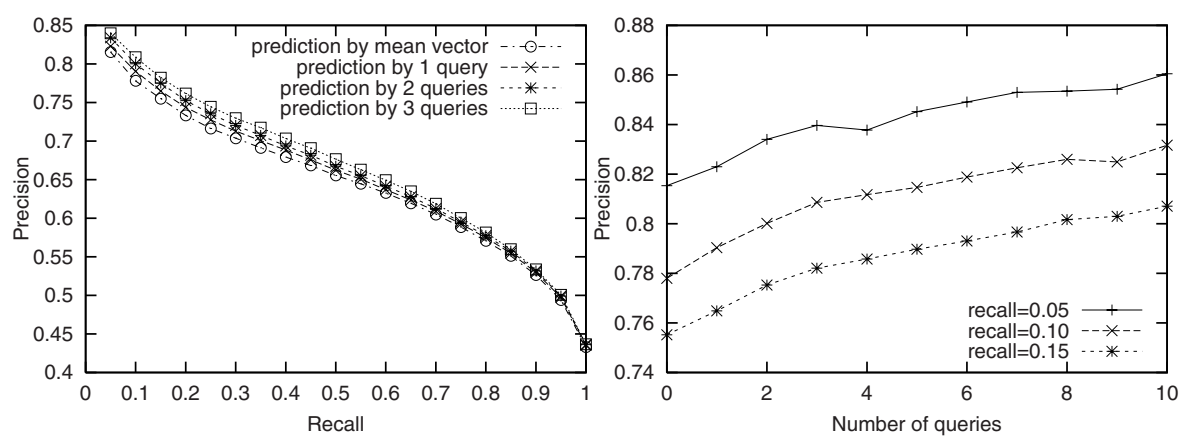

Fig. 4. Left: Recall-precision curves, Right: \#Queries-precision curves

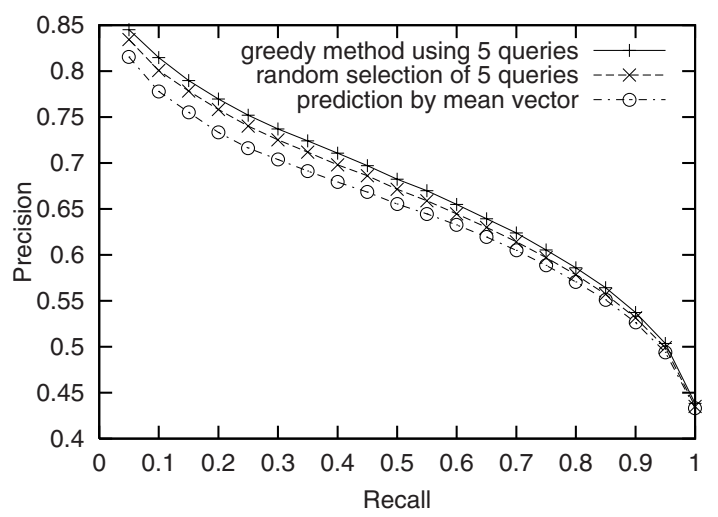

Fig. 5. Comparison with random selection

the right panel of Fig. 4. The rate of improvement decreases when the number of queries is larger than three.

Fig. 5 shows a comparison of the performances of our greedy method and random selection in terms of recall-precision curves. The graph shows the greedy method also outperformed radom selection with respect to this measure.

Are the query items selected by our greedy method effective for the predictions of correlation-based methods? According to the results of our experiments, this is true for the correlation-based method using the item mean. (See Fig. 6.)

\section{Future Work}

The following issues must be considered in future works.

\section{Better estimation of the covariance matrix $R$}

In order to estimate $R$ from a user's preference dataset $X$, we must deal with the problem of missing values. In our experiments, we replaced all missing values with the item mean, but this does not seem to be the best method, 

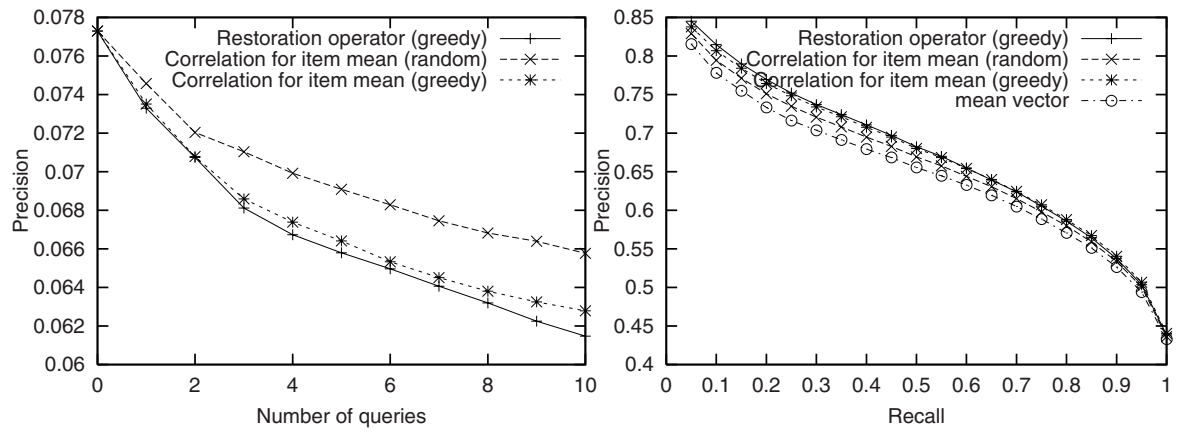

Fig. 6. Left: Learning curves, Right: Recall-precision curves for predictions using 5 queries

especially in the case of large number of missing values. It is possible to estimate the $(i, j)$ entry value $r_{i, j}$ of $R$ from only such vectors $\boldsymbol{x}$ whose $i$ th and $j$ th components are not missing, but the resultant matrix $\hat{R}$ loses the property of non-negative definite, which seems to have a bad influence on the solution.

2. Efficient query item selection method whose solution is closer to the optimal one

According to our experimental results, the performance of our greedy method is only slightly better than that of random selection. The development of a fast algorithm whose solution is closer to the optimal one is therefore needed.

\section{Online query item selection}

The methods proposed in $[3,5]$ are online, that is, the next query item is decided on the basis of answers to the previous queries. Development of such methods in our framework is preferable because there is a possibility that a set of query items more appropriate for the predictions of a user's preference can be selected by using information obtained by the previous queries.

\section{Acknowledgement}

This work was partly supported by a Grant-in-Aid for Scientific Research (B), No. 14380151, from the Japan Society for the Promotion of Science.

\section{References}

1. Andrews, H., Hunt, B.: Digital Image Restoration. Prentice-Hall, Englewood Cliffs, N.J., 1977.

2. Billsus, D., Pazzani, M.: Learning Collaborative Information Filters. Proc. of the 15th International Conference (ICML'98) (1998) 46-54.

3. Boutilier, C. and Zemel, R. S.: Online queries for collaborative filtering. Ninth International Workshop on Artificial Intelligence and Statistics (2002). 
4. Cohn, D., Ghahramani, Z., Jordan, M.: Active Learning with Statistical Models. Journal of Artificial Intelligence Research 4 (1996) 129-145.

5. Dasgupta,S., Lee, W., Long, P.: A Theoretical Analysis of Query Selection for Collaborative Filtering. Machine Learning 51 (2003) 283-298.

6. EachMovie collaborative filtering data set, 1997. research.compaq.com/SRC/eachmovie/.

7. Goldberg, K., Roeder, T., Gupta, D., Perkins, C.: Eigentaste: A Constant Time Collaborative Filtering Algorithm. Information Retrieval Journal 4(2) (2000) 133151.

8. Nakamura, A., Abe, N.: Collaborative Filtering using Weighted Majority Prediction Algorithms. Proc. of 15th International Conference on Machine Learning (1998) 395-403.

9. Ogawa, H., Oja, E.: Projection Filter, Wiener Filter, and Karhunen-Loève Subspaces in Digital Image Restoration. Journal of Mathematical analysis and applications 114 (1986) 37-51.

10. Resnick, P., Iacovou, N., Suchak, M., Bergstom P., Riedl, J.: GroupLens: An Open Architecture for Collaborative Filtering of Netnews. Proc. of CSCW (1994) 175186.

11. Shardanand, U., Maes, P.: Social Information Filtering: Algorithms and Automating "Word of Mouth". Proc. of CHI95 (1995) 210-217. 